\title{
Elbow dysplasia and lesions of the medial coronoid process: correlation between tomographic and arthroscopic findings in thirty cases
}

\author{
A. F. Botazzoli • F. Ferraresi • O. Travetti • \\ F. M. Martini • C. M. Mortellaro • M. Di Giancamillo
}

Published online: 21 August 2008

(C) Springer Science + Business Media B.V. 2008

Keywords Arthroscopy $\cdot$ Dog $\cdot$ Elbow dysplasia $\cdot$ X-ray computerized tomography $(\mathrm{CT})$

\section{Introduction}

Elbow dysplasia is an invalidating pathology with a not yet entirely clarified etiology, that implies a congenital and/or herditary-familiar nature, showing a particularly elevated incidence in some canine breeds (Retrievers, Rottweiler, Newfoundland, Bernese mountain dog etc.) (Olsson 1975; Guthrie and Pidduck 1990). It can be revealed in various forms, (FCP,UAP,OCD,INC) and if it is not precociously diagnosed it also leads, sometimes inevitably, to forms of severe arthrosis (Olsson 1975; Hornof et al. 2000; Kunzel et al. 2004; Blond et al. 2005). The diagnostic procedure consists, besides the clinical examination, of a standardized radiographic examination according to the indications furnished by the International Elbow Working Group (IEWG), that however gives only information on the presence or lack of secondary osteoarthrosic damage (Van Bree and Van Ryssen 1995; Hornof et al. 2000; Blond et al. 2005). For this reason, in the last decade the use of second level imaging techniques as CT and arthroscopy, which has the undeniable advantage to offer both diagnostic and therapeutic options, has increased (Van Bree and Van Ryssen 1995; Bardet 1997; Van Ryssen and Van Bree 1997; Hornof et al. 2000; Reichele et al. 2000; De Rycke et al. 2002; Rovesti 2002; Schultz et al. 2004; Blond et al. 2005; Martini 2006). The present works is to be considered as a retrospective investigation performed on 30 articulations affected by elbow dysplasia to find a possible correlation between CT and arthroscopic findings of different nature and degree, contextually analyzing the possibilities and limits of each technique.

\footnotetext{
A. F. Botazzoli $(\bowtie) \cdot$ F. Ferraresi $\cdot$ O. Travetti $\cdot$ C. M. Mortellaro $\cdot$ M. Di Giancamillo Dipartimento Scienze Cliniche Veterinarie, Sezione di Radiologia veterinaria clinica e sperimentale, Facoltà di Medicina Veterinaria, Via Celoria 10, 20133 Milano, Italy e-mail: Andrea.Botazzoli@unimi.it

F. M. Martini

Dipartimento di Salute Animale, Facoltà di Medicina Veterinaria, Parma, Italy
} 


\section{Materials and methods}

Thirty cubital joints belonging to 16 dogs with clinical and radiological signs of elbow dsysplasia were examined. Each patient, maintained under general anesthesia, was submitted to CT examination using a fourth generation scanner mod.PQ2000S (Philips S. p.A.) according to the following protocol: thick/index $1 \mathrm{~mm}$, perpendicular to radius and ulna, $120 \mathrm{kV}, 125-175 \mathrm{~mA}$, Scan Time $1 \mathrm{sec}$, high definition convolution filters, visualization parameters for the study of hard tissues. Each joint was acquired singly. Each study was subsequently submitted to multiplanar reconstructions (MPR) on sagittal and dorsal planes. The observed lesions were recorded according to a modified version of the classification proposed by Reichle et al. (Reichle et al. 2000). A rigid endoscope with 2.4 or $2.7 \mathrm{~mm}$ diameter, $30^{\circ}$ lens angle and a 3.2 or $4 \mathrm{~mm}$ diameter cannula respectively, were used for the arthroscopic examination. A medial approach was used, and the alterations were classified according to a modification of a system proposed by Van Ryssen and Van Bree (1997) and Schultz et al. (2004). Where possible the arthroscopic examination was performed immediately at the end of the CT investigation to optimize the length of the study in an single anestesiologic session.

\section{Results}

The thirty articulations belonged to 7 different breeds, (4 Labrador R., 3 Bernese mountain dogs, 3 Rottweilers, 1 German Shepherd, 1 Corso dog, 1 English Bulldog and 3 Mixed). 4 females and 12 males between 7 and 24 months of age (average 10.9 months, median 8.5 months). The median weight was of $31.5 \mathrm{Kg}$. The CT showed 22 cases of FCP (8 type I, 5 type II, 2 type III, 1 plurifragmentation and 6 fissurations). In 4 cases the PCM was hypodense and with anomalies of form, while in 2 cases only morphological anomalies were present, 1 case presented osteophytes on the border of the PCM and 1 appeared normal. Besides the evaluation of the PCM the CT allowed observation of 3 cases of OCD/ $\mathrm{KL}, 1$ case of UAP and 1 of ossification of the tendons of the flexor muscles (OTMF). Eighteen INC (17 cases of "long ulna" and 1 of "short ulna") were also observed. The arthroscopic examination identified a total of 19 FCP (9 type I, 2 type II, 2 type III, 2 plurifragmentation and 4 cases of deep fissurations). In 2 cases superficial fissurations were observed, while in another 6 cases cartilage fibrillation was diagnosed. In 2 joints the MPC appeared normal. The INC observed were 11 in total (8 long ulna and 3 short ulna).

\section{Discussion}

The observation and analysis of the data has pointed out a discrepancy between the lesions seen in CT and those observed with the arthroscopy. In general considering the lesions of the MCP, the CT had the tendency to overestimate morphostructural alterations, in particular concerning FCP type II. This is explicable taking into consideration that the radial incisure of the ulna is a precocious site of structural alteration in CT and the morphostructural anomalies observed (hypodensites, irregularity, geodi, ostephytes, etc) can induce to the formulation of false positives. We also have to remember that for the peculiar spatial orientation of the PCM, the orthogonality between object and scans cannot be maintained for the whole phase of the acquisition and therefore it is possible that under critical conditions the machine can produce small artifacts (Van Bree and Van Ryssen 1995; Reichle et al. 2000; Rovesti 2002; Blond et al. 2005). It is difficult for the CT to establish 
the entity of alterations of the cartilage, an aspect which is more easily observable with the arthroscopic lenses. Contrarily concerning the alterations of the subchondral bone it is a different case, where these are not visible with the arthroscopic examination but exclusively by tomographic investigation (Van Bree and Van Ryssen 1995; Reichle et al. 2000). On the contrary CT does not allow differentiation between OCD and KL as this is possible only with arthroscopy. Also, considering the evaluation of INC, there were important differences. In this case we believe that arthroscopy underestimates INC whih it is more difficult to detect for the loss of the normal anatomical reference points caused by the distorsions resulting from the introduction of the scope inside the articulation (Reichele 1999) and it is also completely operator-dependent. Contrarily, the MPR reconstructions in CT performed at the level of the LCP allow standardization of the methodology and attainment of more constant parameters (Gemmil et al. 2005). This work has underlined the fact that the two imaging techniques, however, does not have to be considered in competition, but as complementary, and it suggests further evaluations of the fissurations of the MCP by CT, as a possible technique for the precocious diagnosis of FCP.

\section{References}

Bardet J.F., 1997. Arthroscopy of the elbow in dogs Part I. Veterinary and comparative orthopaedics and traumatology, 10(1-5), 60-66.

Blond L., Dupuis J., Beauregard G., Breton L., Moreau M., 2005. Sensitivity and specificity of radiographic detection of canine elbow incongruence in an in vitro model. Veterinary Radiology and Ultrasound, 46, $210-216$.

De Rycke L.M., Gielen I.M., van Bree H., Simoens P.J., 2002. Computed tomography of the elbow joint in clinically normal dogs American Journal Veterinary Research, 63, 1400-1406.

Gemmil T.J., Mellor D.J., Clements D.N., Clarke S.P., Farrell M., Bennett D., Carmichael S., 2005. Evaluation of elbow incongruency using reconstructed CT in dogs suffering fragmented coronoid process. Journal of Small Animal Practice, 46, 327-333.

Guthrie S. and Pidduck H.J., 1990. Hereitability for elbow osteochondrosis within a closed population of dogs. Journal of Small Animal Practice, 31, 93-96.

Hornof W.J., Wind A.P., Wallack S.T., Schulz K.S., 2000. Canine elbow displasya: the early radiographic detection of fragmentation of the coronoid process. North America Small Animal Practice, 30, 257-266.

Kunzel W., Breit S., Probst A., 2004. The subchondral split line patterns of medial coronoid process in the canine ulnae. Anatomia, histologia, embryologia, 33, 339-343.

Martini F.M., 2006. Patologie articolari nel cane e nel gatto. Poletto Editore: Milano.

Olsson S.E., 1975. Lameness in the dog: a review of lesions causing osteoarthrosis of the shoulder, elbow, hip, stifle and hock joint. American Animal Hospital Association Proceedings, 1, 363.

Reichele J.L., 1999. The Elbow. Clinical techniques in small animal practice, 14(3), 177-186.

Reichle J.K., Park R.D., Bahr A.M., 2000. Computed tomographic findings of dogs with cubital joint lameness. Veterinary Radiology and Ultrasound, 41, 125-130.

Rovesti G.L., 2002. The use of computed tomography in the diagnostic protocol of the elbow in the dog: 24 cases. Veterinary and comparative orthopaedics and traumatology, 15, 35-43.

Schultz K., Holsworth I.G., Hornof W.J., 2004. Self-retainig braces for canine arthroscopy. Veterinary Surgery, 33, 77-82.

Van Bree H. and Van Ryssen B., 1995. Imaging the canine elbow: radiography,computed tomography and arthroscopy. Veterinary Annual, 35, 118-129.

Van Ryssen B. and Van Bree H., 1997. Arthroscopic findings in 100 dogs with elbow lameness. Veterinary Record, 140, 360-362. 